\title{
Waveguide properties recovered from shallow diffractions in common offset GPR
}

\author{
Elmar Strobach, ${ }^{1}$ Brett D. Harris, ${ }^{1}$ J. Christian Dupuis, ${ }^{1}$ and Anton W. Kepic ${ }^{1}$ \\ Received 16 May 2012; revised 23 October 2012; accepted 25 October 2012; published 17 January 2013.
}

[1] Near-surface heterogeneities produce diffractions in common offset ground-penetrating radar (GPR) data from the Gnangara Groundwater Mound, north of Perth, Western

Australia. These diffracted wavefields can be enhanced and show a dispersion pattern if they propagate along a waveguide caused by a low velocity surface layer, such as moist sand on top of dry sand. Until now, GPR waveguide dispersion has been analyzed and inverted using common midpoint data. Using numerical modeling, we demonstrate that the same dispersion information can also be recovered from a diffracted electromagnetic wavefield recorded with common offset geometry. Frequency-slowness analysis of shallow diffractions in common offset GPR field data reveals high resolution dispersion curves. Inverting picked dispersion maxima to modeled curves (i.e., modal wave propagation in waveguide layer) allows estimation of waveguide height and velocities of waveguide and the underlying material. Data analysis in the frequency-wavenumber domain provides an alternative technique for extracting dispersion curves. Preliminary results validate this approach, which could be favorable in large-scale applications due to minimal processing requirement and inherent yet adjustable spatial averaging. The differences between waveguide parameters recovered from two surveys appear to be consistent with seasonal changes in moisture content and lateral changes due to variations in depositional environment. Our approach presents a new method to quantify the shallow dielectric permittivity structure of the subsurface from common offset gathers - the most commonly acquired type of GPR data. Potential applications of this method include estimation of shallow moisture distribution, early target identification for unexploded ordnance (UXO) detection, concrete slab characterization, pedological investigations, or planetary exploration.

Citation: Strobach, E., B. D. Harris, J. C. Dupuis, and A. W. Kepic (2013), Waveguide properties recovered from shallow diffractions in common offset GPR, J. Geophys. Res. Solid Earth, 118, 39-50, doi:10.1029/2012JB009448.

\section{Introduction}

[2] The near-surface moisture distribution is of great interest for several Earth-related disciplines such as agriculture, plant ecology, civil engineering, soil science, and hydrogeology. Because the dielectric permittivity is largely controlled by water content, measurements of the relative dielectric permittivity of the medium can provide information about soil moisture [Huisman et al., 2003]. A ground-penetrating radar (GPR) system consists of an arrangement of antennas that can emit and receive electromagnetic pulses in the radar frequency range of $1 \mathrm{MHz}$ to several gigahertz. These pulses propagate through low-loss materials until they are reflected or diffracted by interfaces and objects that exhibit contrasts

\footnotetext{
${ }^{1}$ Department of Exploration Geophysics, Curtin University, Kensington, Australia.

Corresponding author: E. Strobach, Department of Exploration Geophysics, Curtin University, ARRC Building, H Block, Level 4, 26 Dick Perry Ave., 6151 Kensington, Perth, Western Australia, Australia.

(elmar.strobach@postgrad.curtin.edu.au)

(C)2012. American Geophysical Union. All Rights Reserved. 2169-9313/13/2012JB009448
}

in conductivity and electric permittivity. The propagation velocity is inversely proportional to the square-root of the relative dielectric permittivity and hence moisture content can be inferred [Huisman et al., 2003]. Several authors have examined methods for quantifying very near-surface dielectric permittivity from GPR common offset (CO) gather (COG) based on velocity or reflectivity determination. A COG represents point measurements at a constant spatial sampling interval. With bistatic systems, which are composed of separate transmit and receive antennas, a COG is acquired with a constant antenna separation (hence common offset). In the case of a monostatic system, where the transmit and receive antenna are the same, the offset is zero.

[3] The most common type of GPR systems are based on ground-coupled and air-launched techniques [Pettinelli et al., 2007; Lambot et al., 2006; Grote et al., 2010; Huisman et al., 2003]. These approaches, however, tend to lack depth of penetration (e.g., air-launched) or seldom provide a conclusive layered Earth model (e.g., bistatic constant offset). Multioffset methods such as common midpoint (CMP-variable antenna separation) or common shotpoint (CSP) gather provide an alternative method of determining dielectric properties of the shallow subsurface by analyzing the arrival times of the 

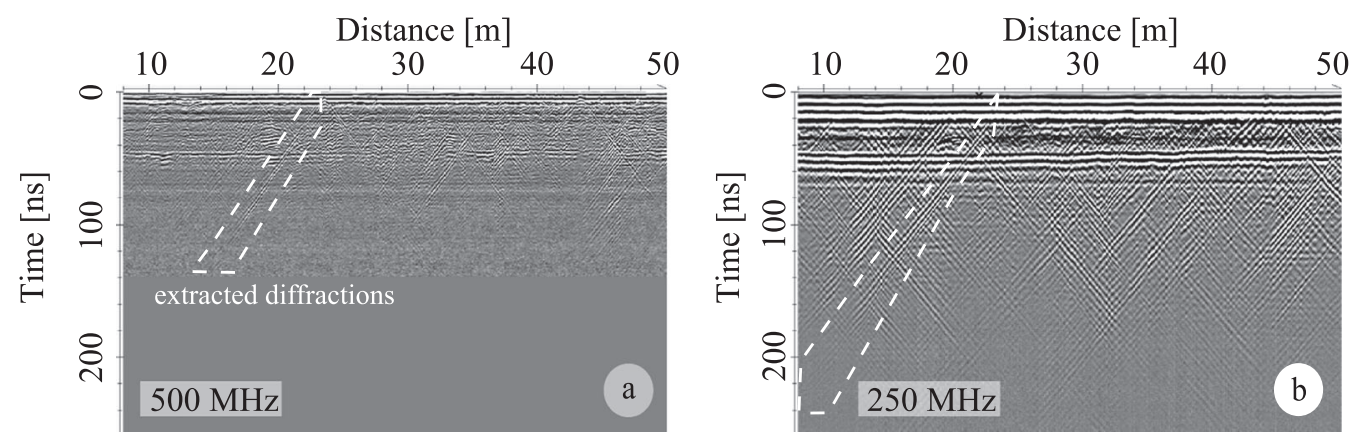

Figure 1. Common offset field example from the May 2011 survey showing argyle-like pattern created by dispersive diffraction hyperbolae originating from shallow diffractors within a low-velocity waveguide (western Lexia West site). Diffraction onsets are first seen from up to $14 \mathrm{~m}$ ahead of the diffractor for the $250 \mathrm{MHz}$ data in Figure 1b, which can be explained by low-loss propagation of waveguide modes.

direct groundwave. Alternatively, a one-dimensional velocity model can be derived from reflections using semblance analysis. The length of time required to acquire CMP and CSP gathers with a GPR system that consists of a single transmit and receive antenna pair, typical of most commercial systems available at present, precludes its widespread use. Also, several of the bistatic systems commercially available are prescriptive about the antenna spacing that can be used during acquisition and thus making acquisition of multioffset data impossible.

[4] After collecting and analyzing many hundreds of kilometers of GPR data with a bistatic COG configuration, we noticed that numerous diffractions in the data, originating from roots, stones, and other subwavelength unconformities, had signals enhanced by waveguide phenomena (e.g., Figure 1). Because these diffraction signals are measurable from significant distances from the diffraction location, they can be analyzed with a multioffset data analysis approach, as used in the CMP or CSP method. However, to do this requires an understanding of how the diffracted COG-GPR signal behaves in near-surface waveguides, which we investigate in section 2.

[5] Waveguide effects in GPR data were first described and quantified by Arcone [1984] who applied modal propagation theory to explain the phenomenon. Subsequent authors refined the approach by introducing inversion schemes to obtain geological models [Busch et al., 2012; Strobbia and Cassiani, 2007; Van der Kruk, 2006; Van der Kruk et al., 2006, 2009a, 2010]. Until now, the use of the waveguide dispersion observed in the diffraction hyperbola of COGs to compute dielectric properties has largely been ignored, or unreported. Arcone et al. [2003] observed these signals as "diagonal streaks", which they identified as backscattered modes travelling in a surface layer. From the slope of these streaks they concluded that they were waveguide modes excited by diffractors. Cassiani et al. [2006] noticed similar diffractions in their GPR data. Van der Kruk et al. [2010] observed that these diffractions were dispersive and, in their example, originated from shallow boulders. Steelman et al. [2010] identified dispersive scattering events in their $\mathrm{CO}$ data and say that "inverse analysis [...] should yield physical property measurements analogous to that obtained from CMP soundings."

[6] In this work, we show, using finite-difference timedomain (FDTD) modeling, that diffraction hyperbolae observed in CO-GPR data, where a shallow waveguide exists, exhibit the same dispersion characteristics as would be recovered from the CMP or CSP survey geometries. We then demonstrate that shallow soil properties can be derived from dispersive diffractions in $\mathrm{CO}$ data acquired at a sandy soil environment (e.g., Figure 1). Dispersion curves are extracted from (i) frequency-slowness domain (i.e., slant stack, Park et al. [1998]) and (ii) frequency-wavenumber spectrum. Waveguide parameters are estimated by inversion and the parameter space is investigated. Observed curves are matched using modal wave propagation in a low-velocity waveguide layer model [Van der Kruk, 2006].

\section{Synthetic Example}

[7] Dispersion images in the frequency-slowness domain can be calculated from radargrams with the method described by Park et al. [1998] and adapted by Van der Kruk [2006]. By picking maxima of fundamental and higher modes from trace-normalized dispersion images, we can obtain dispersion curves. In the following synthetic example we compare the dispersion images for CSP and CO configurations and show that dispersion curves recovered from (i) the direct and diffracted wavefield and (ii) sampled with a $\mathrm{CO}$ and CSP geometry, are identical after two-way travel time adjustment. We will discuss some characteristics of the dispersion images obtained from diffractions sampled with COGs.

\subsection{Forward Modeling}

[8] The full electromagnetic wavefields are simulated by forward modeling using a two-dimensional FDTD scheme included in the software package ReflexW [Sandmeier, 2012]. We compare results from (i) the direct CSP wavefield with the CSP diffracted wavefield and (ii) the diffracted wavefield sampled as COG with the CSP results. Our FDTD analysis is for the transverse electric TE $\left(E_{y^{-}}\right)$field components. The model consists of three layers representing a waveguide: (i) an air layer (highest velocity) underlain by (ii) a low-velocity horizon (waveguide-layer), which is itself underlain by (iii) a high-velocity layer (Figures $2 \mathrm{a}$ and $2 \mathrm{~d}$ ). We embed a diffractor in the low-velocity layer (layer 2) to create the diffracted energy. The diffractor is a circular area with a diameter of $0.4 \mathrm{~m}$ and a relative dielectric permittivity of 25. This is significantly higher than the low-velocity layer, which is given a dielectric permittivity of 5.5 yielding 

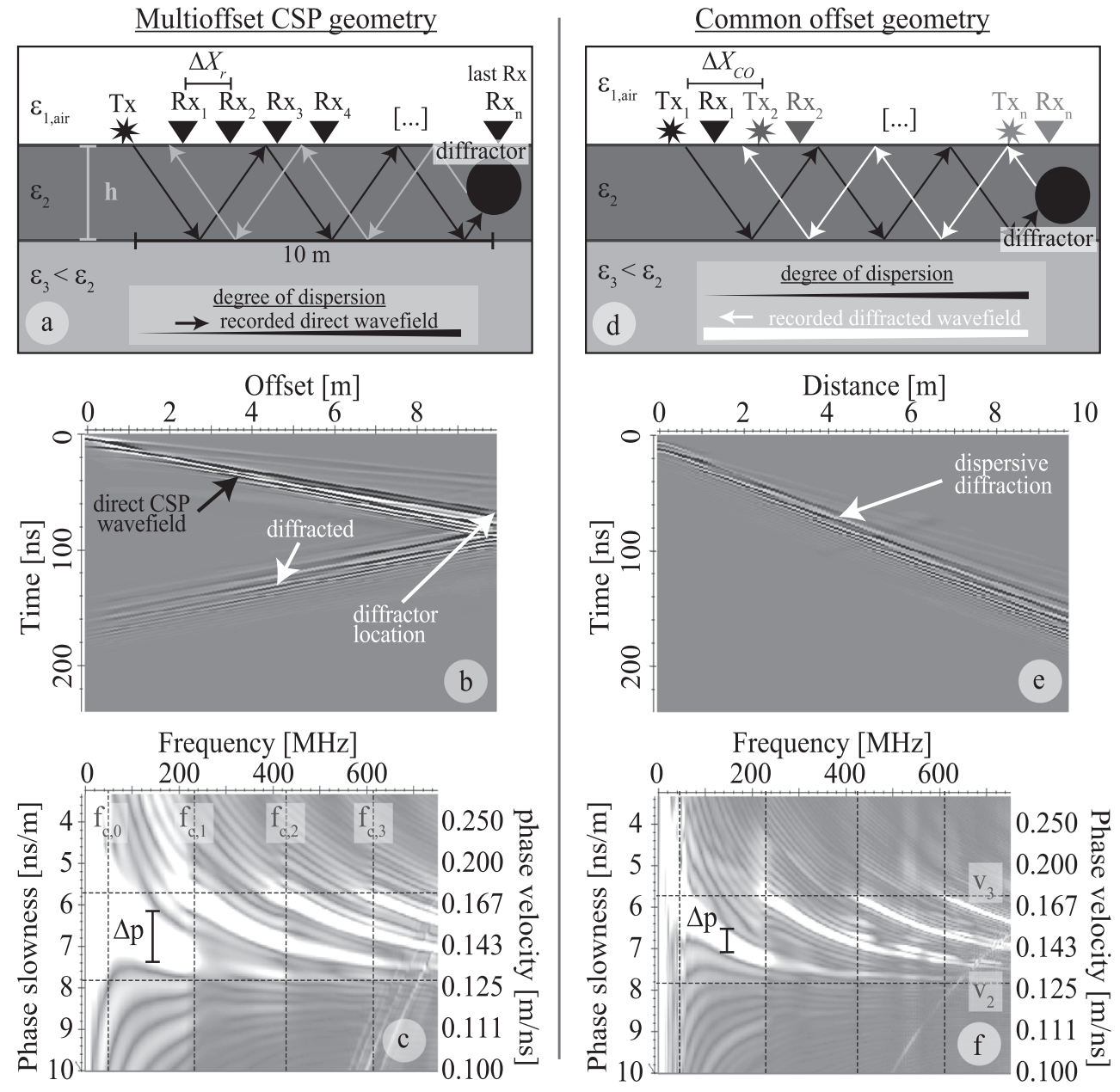

Figure 2. Waveguide model used for FDTD modeling corresponding to (a) a common shot point and (d) a common offset geometry. Shot (transmitter Tx) and receiver (Rx) positions are shown as stars and triangles, respectively. Grayscale density represents relative dielectric permittivity with white being the lowest (i.e., $\varepsilon_{\text {air }}=1$ ) and black (diffractor) the highest value (i.e., $\varepsilon_{\text {diff }}=25$ ). Ray-paths of a direct and diffracted phase travelling in the waveguide layer are displayed in black and gray/white, respectively. For the CSP geometry, dispersion can be derived from waves either following the black (direct) or gray (diffracted) ray-paths, while the CO mode samples white rays (diffracted). Synthetic (b) common shotpoint and (e) common offset gathers. Their corresponding dispersion images are shown in Figures $2 \mathrm{c}$ and $2 \mathrm{f}$. FDTD modeling parameters are given in Table 1. Note the increased resolution $\Delta p$ (length of black bars) of the slowness maxima obtained in Figure $2 \mathrm{f}$ due to the increased effective spread length. Phase slownesses in Figure $2 \mathrm{f}$ have been calculated with two-way travel time. All plots are trace normalized.

a strong impedance contrast. A high permittivity circular object could, for example, represent a water saturated tree root. Time and space discretization (i.e., $\Delta t$ and $\Delta x$ ) for the FDTD modeling was set to $0.04 \mathrm{~ns}$ and $0.025 \mathrm{~m}$, respectively (see Table 1). This discretization is sufficiently small to prevent numerical dispersion in time and space. Model boundaries were set to the largest exponential absorbing boundaries possible in ReflexW. Modeling parameters for the synthetic study are summarized in Table 1.

[9] The ground-arrivals in the modeled radargram of the CSP gather exhibit characteristics of a dispersive direct groundwave (Figure 2b). Note the diffracted, left dipping wavefield in Figure $2 \mathrm{~b}$ that originates at a position of $10 \mathrm{~m}$ (i.e., diffractor location) at approximately $50 \mathrm{~ns}$. Figure $2 \mathrm{e}$ shows the diffracted wavefield sampled with a CO survey configuration, which we want to compare with the CSP geometry results. Note that the travel-time curve of the diffracted $\mathrm{CO}$ arrivals is linear, not hyperbolic. The presence of a waveguide results in mainly lateral wave propagation and hence the moveout of the diffraction arrivals appear as if the diffractor is at "zero" depth. This observation means that single-frequency phase velocity $v_{\mathrm{p}}$ can be approximated from the slope of those diffraction events after applying narrow bandpass filters [Van der Kruk et al., 2009b]. The moveout function is linear and can be written as $v_{\mathrm{p}}=2 \mathrm{~d} x / \mathrm{d} t$. Hence, the characteristics of shallow diffracted wavefields are inherently similar to direct ground-arrivals observed on CMP or CSP gathers with the exception of a factor of two. Figure 2e shows the synthetic COG with diffracted arrivals to the right of the diffractor. Traces have been re-sorted so that the diffractor is now located at $x=0$. Dispersion images of both 
Table 1. Waveguide, Underlying Layer, and Diffractor Parameters Used for the Synthetic Study. Cutoff Frequencies for Fundamental $\left(f_{\mathrm{c}, 0}\right)$ and Higher Modes $\left(f_{\mathrm{c}, m}\right)$ are Calculated From Waveguide Parameters Using Equation (1)

\begin{tabular}{lccc}
\hline & $v[\mathrm{~m} / \mathrm{ns}]$ & $\varepsilon_{r}$ & $h[\mathrm{~m}]$ \\
\hline FDTD layer 1 & 0.300 & 1 & 2.00 \\
FDTD layer 2 & 0.128 & 5.5 & 0.50 \\
FDTD layer 3 & 0.173 & 3 & 3.00 \\
FDTD diffractor & 0.060 & 25 & 0.40 \\
$f_{\mathrm{c}, 0}: 44 f_{\mathrm{c}, 1}: 234 f_{\mathrm{c}, 2}: 424 f_{\mathrm{c}, 3}: 613$ & & \\
FD $\Delta t: 0.04 \mathrm{~ns}$ & & \multicolumn{2}{c}{ Receiver $\Delta X_{r}: 0.1 \mathrm{~m}$} \\
FD $\Delta x: 0.025 \mathrm{~m}$ & \multicolumn{2}{c}{ CO shot $\Delta X_{\mathrm{CO}}: 0.1 \mathrm{~m}$} \\
\hline
\end{tabular}

wavefields and sampling methods reveal very similar dispersion images (Figures 2c and 2f). Picked maxima of the dispersion images from both survey geometries are displayed in Figure 3. In addition, the dispersion curve recovered from the CSP diffracted wavefield (dispersion image not shown here) is also shown in Figure 3. The critical frequencies $f_{\mathrm{c}, m}$ for mode $m$ displayed as dashed vertical lines in Figures $2 \mathrm{c}$, $2 \mathrm{f}$, and 3 (values are listed in Table 1) were calculated using the equation given by Van der Kruk [2006] for the TE modes

$$
f_{c}=\frac{c \cdot\left[2 \pi m+\phi^{\mathrm{TE}}\left(\theta_{\mathrm{c}, 23}\right)\right]}{4 \pi h \sqrt{\varepsilon_{2}-\varepsilon_{3}}}
$$

with $\phi^{\mathrm{TE}}$ the phase component for the critical angle $\theta_{\mathrm{c}, 23}$ for mode propagation that can be written as

$$
\phi^{\mathrm{TE}}\left(\theta_{\mathrm{c}, 23}\right)=\tan ^{-1}\left(\frac{2 \sqrt{\varepsilon_{2}-\varepsilon_{3}} \sqrt{\varepsilon_{2}-\varepsilon_{\mathrm{air}}}}{\varepsilon_{\mathrm{air}}+\varepsilon_{2}-2 \varepsilon_{3}}\right)
$$

\subsection{Characteristics of Synthetic Dispersion Images From Common Offset Gathers}

[10] The CO dispersion curves provide an apparent higher resolution of the slowness compared to the CSP gather (see for example Figures 2c vs. 2f) after correcting for two-way travel. The slowness resolution for a CSP geometry is given by the half distance between neighboring minima (half the width of slowness maxima) $\Delta p=2 \pi / \omega L$, where $L$ is the spread length [Forbriger, 2003]. Thus, the mathematical explanation for the apparent increase in resolution for $\mathrm{CO}$ geometry is the two-way time correction that multiplies $\Delta p$ with a factor 0.5 . The physical reason can be explained by assuming that the dispersive wavefield is back-scattered by the diffractor and continues to disperse along the waveguide as indicated in Figure $2 \mathrm{~d}$. The wavefield therefore effectively covers twice the distance in the waveguide. This means that the diffractor can be considered as an equivalent source emitting a dispersive wavefield, sharing some characteristics of a time-varying frequency sweep that has low frequencies at the beginning and higher frequencies at later times. Thus, for the CO field configuration, the phase velocities at each frequency have greater travel time to separate, and consequently the apparent resolution of the dispersion images can appear superior compared to a dispersion image of a CSP gather with equivalent maximum spread length. We determined $\Delta p$ from the dispersion images of synthetic two-way time corrected $\mathrm{CO}$ diffraction hyperbola at different frequencies and obtained estimates for the spread length between 17.8 and $19.5 \mathrm{~m}$. The farthest $\mathrm{CO}$ pair is $10 \mathrm{~m}$ away from the diffractor. The effective spread length $L_{\text {eff }}$ for CO diffraction hyperbolae is, as predicted, almost doubled.

[11] The additional travel distance in the waveguide means that signal-to-noise ratio of the dispersion image is potentially reduced and the aliasing frequency and consequently the aliasing velocity are increased compared to an equivalent offset CSP or CMP gather. Spatial aliasing is especially prevalent in slow waveguides (e.g., water above ice) and for frequencies at the higher end of the radar band. Additional problems can occur when other wavefields with similar moveout such as diffractions from deeper targets cause interference. For example, deeper diffractions with dispersive diffraction legs have the potential to make $\mathrm{CO}$ dispersion analysis more complicated. Note that horizontal reflectors such as a water table would tend to be more problematic in multioffset dispersion analysis compared with $\mathrm{CO}$ dispersion analysis because in $\mathrm{CO}$ data it produces zero slowness in the frequency-slowness domain (i.e., slope of flat reflection in $\mathrm{CO}$ profile is zero, hence velocity goes toward infinity). Steeply dipping reflectors may also cause interference when interpreting dispersive diffractions in the frequency-slowness domain. The influence of other wavefields on dispersion images is certainly an important consideration and may form a basis for future research on analysis of dispersive diffractions.

\section{Field Experiments and Results}

\subsection{Field Observations}

[12] In this section, we show that the dispersion analysis of diffracted wavefields described in the previous section

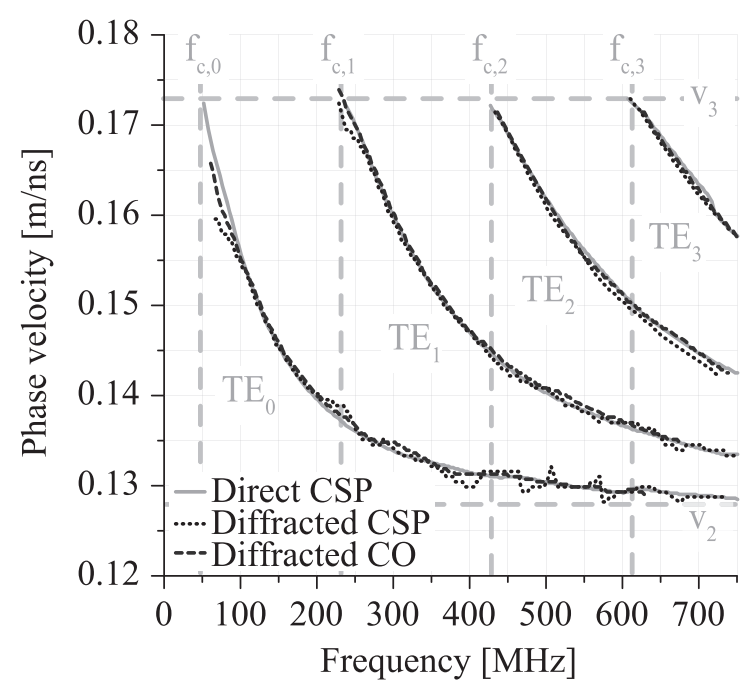

Figure 3. Dispersion curves derived from FDTD modeling corresponding to direct waves (solid lines), diffracted wavefields sampled with CSP geometry (dotted lines) and with $\mathrm{CO}$ geometry (dashed lines). A good match between dispersion curves is observed for fundamental and higher order modes. Minor variations are due to numerical inaccuracies from converting into frequency-slowness domain. 
can be applied to two CO data sets collected on the Gnangara Mound, north of Perth, Western Australia in May and September 2011. The data sets were acquired with the Mala ProEx 2 system with 250 and $500 \mathrm{MHz}$ shielded antennas. Measurements were triggered at $0.025 \mathrm{~m}$ intervals using an encoder wheel. Field measurements on 6 May 2011 were acquired at Lexia West after $18 \mathrm{~mm}$ of precipitation was recorded at a close-by climate observatory over the preceding week (http://www.bom.gov.au/climate/data/). Shallow infiltration after rainfall resulted in a layer of moist sand trapped between an air layer above and dry sand below. In the time between the May and September experiments, cumulative rainfall of approximately $550 \mathrm{~mm}$ increased the background moisture content [Strobach et al., 2012b]. The measurements on 21 September 2011 are from the northern Yeal area and were also preceded by a period of rainfall with approximately $35 \mathrm{~mm}$ precipitation starting on 16 September after 12 days of dry and sunny conditions. The May 2011 data set is taken from a $100 \mathrm{~m}$ long profile, while the September 2011 data are extracted from a larger-scale investigation spanning approximately $5 \mathrm{~km}$. Both sites are situated on the Bassendean Sand formation, which is dominated by vegetated dunes with wetlands common at the depressions [Davidson, 1995]. The shallow material at both areas consists of an A-horizon, which is a mixture of leached quartz sand and dispersed organic material. Below is a leached Ehorizon typical for podosolic soils on the Gnangara Mound [Prankongkep et al., 2010]. It is characterized by well sorted medium grained quartz sand of pale white to gray color. The September profile intersected a wetland that contains peatlike material in the A-horizon.

[13] Figure 1 shows CO radargrams collected in May 2011 with the $500 \mathrm{MHz}$ (Figure 1a) and $250 \mathrm{MHz}$ (Figure 1b) antennae. The sections are characterized by numerous lines running diagonal through the radargram with positive and negative slopes creating an argyle pattern. We interpret those events to originate from diffractors embedded in a shallow waveguide. Although radiation pattern of modern GPR systems emit some energy forward (i.e., lateral waves [Chen, 2012]), a strong response from shallow diffractors is not expected to be detectable several wavelength ahead of the target in low-loss dielectric environment. A waveguide, however, captures EM energy and facilitates lossless wave propagation. Diffracted energy can then have equivalent or higher amplitudes at late times compared to reflected waves from greater depth. The apex of those diffraction hyperbolae (i.e., where the straight lines meet) is close to zero time, which indicates very shallow diffractors. Tree roots, buried rocks, patches of hydrophobic sand, or boulders [Van der Kruk et al., 2010] are all known and identified potential sources of shallow diffractors. We investigated soil conditions in the field and identified elevated moisture within the first approximately $30 \mathrm{~cm}$. Shallow diffractions appear to be correlated with vegetation and shallow excavation revealed dry spots associated with grasstree roots (Xanthorrhoea). We hypothesize that dry spots within a wet soil might reasonably function as diffractors for the electromagnetic waves.

[14] Common offset data in Figure 1b show diffracted energy at up to $15 \mathrm{~m}$ distance from the diffractor at arrival times of up to $240 \mathrm{~ns}$ for the $250 \mathrm{MHz}$ data. The horizontal reflector at approximately $45 \mathrm{~ns}$ is interpreted as the reflection from the water table or the capillary fringe. Depth to water was measured at a close-by well (Lexia West) to be $3.9 \mathrm{~m}$ below surface level. This leads to an average interval velocity of approximately $0.17 \mathrm{~m} / \mathrm{ns}$ for the sand above the water table.

[15] The September $250 \mathrm{MHz}$ data from northern Yeal (Figure 4) showed two types of dispersive diffractions. The first type represents strong dispersive diffractions that can be observed up to $18 \mathrm{~m}$ ahead of the diffractor at arrival times of $300 \mathrm{~ns}$. These are denoted as September I (SIaSId) in Figures $4 d$ and $4 \mathrm{e}$ and is associated with clean sands below the vegetated dunes. The second type, called September II (SIIa and SIIb), is associated with the lower wetlands spanning the first $600 \mathrm{~m}$ of the profile shown in Figure 4a. Diffractions SIIa and SIIb in Figures $4 b$ and $4 c$, respectively, are weak and detectable up to $5 \mathrm{~m}$ (120 ns) and $8 \mathrm{~m}$ (200 ns) ahead of the diffractor, respectively. The section of the radargram across the wetland area shows increased attenuation, which is likely to be a consequence of an increased fraction percent of damp loamy sand and a peat-like surface layer. Two-way travel time to the water table in the Yeal area is dependent on topography and can vary between 40 and 200 ns. Exact water table position is not known here; however, it is a relatively flat interface. The interval velocity required to create a flat water table reflection after topographic correction is approximately $0.13 \mathrm{~m} / \mathrm{ns}$. This value of $0.13 \mathrm{~m} / \mathrm{ns}$ is generally consistent with velocities estimated from deeper hyperbolic diffractions that originate close to the water table. Note that the step in the water table level between 1300 and $1800 \mathrm{~m}$ is currently interpreted as a hydrologic transition and not due to lateral velocity variations.

\subsection{Data Processing}

[16] We extracted single diffraction legs from the COGs shown in Figures 1 and 4. The diffraction legs are marked with white lines in both figures. Single diffractions were chosen for dispersion analysis based on their dispersive character, signal-to-noise ratio, and the absence of interfering wavefields. Further processing was performed in ReflexW and included a DC-shift removal (subtraction of average trace amplitudes within a late time window) and application of a time-dependent gain function calculated from the mean amplitude decay of all traces. We then muted the area above and below the diffraction leg with a cosine taper window of $5 \mathrm{~ns}$. Processed data are shown in Figures 4b, 4c, $4 \mathrm{~d}, 4 \mathrm{e}, 5 \mathrm{a}, 5 \mathrm{c}$, and $5 \mathrm{e}$. This simple processing flow was designed to minimize the impact of the processing on the phase characteristics of the signals. Phase slowness spectra were then calculated using a slant stack as described by Park et al. [1998] and shown in Figures $4 \mathrm{f}-4 \mathrm{j}$ and Figures 5b, 5d, and $5 \mathrm{f}$. Dispersion curves are then extracted by picking maxima from the dispersion images.

[17] An interesting alternative to calculating frequencyslowness distribution, as above, is to perform dispersion analysis in the frequency-wavenumber ( $f k$-) domain. An example of $f k$-analysis is given for the May $250 \mathrm{MHz}$ data set in Figure 6 . The $f k$-spectrum makes it possible to identify energy associated with the moveout of the diffractions due to characteristic $f k$-slope. Phase velocity for the CO data is calculated from frequency and wave number picks 


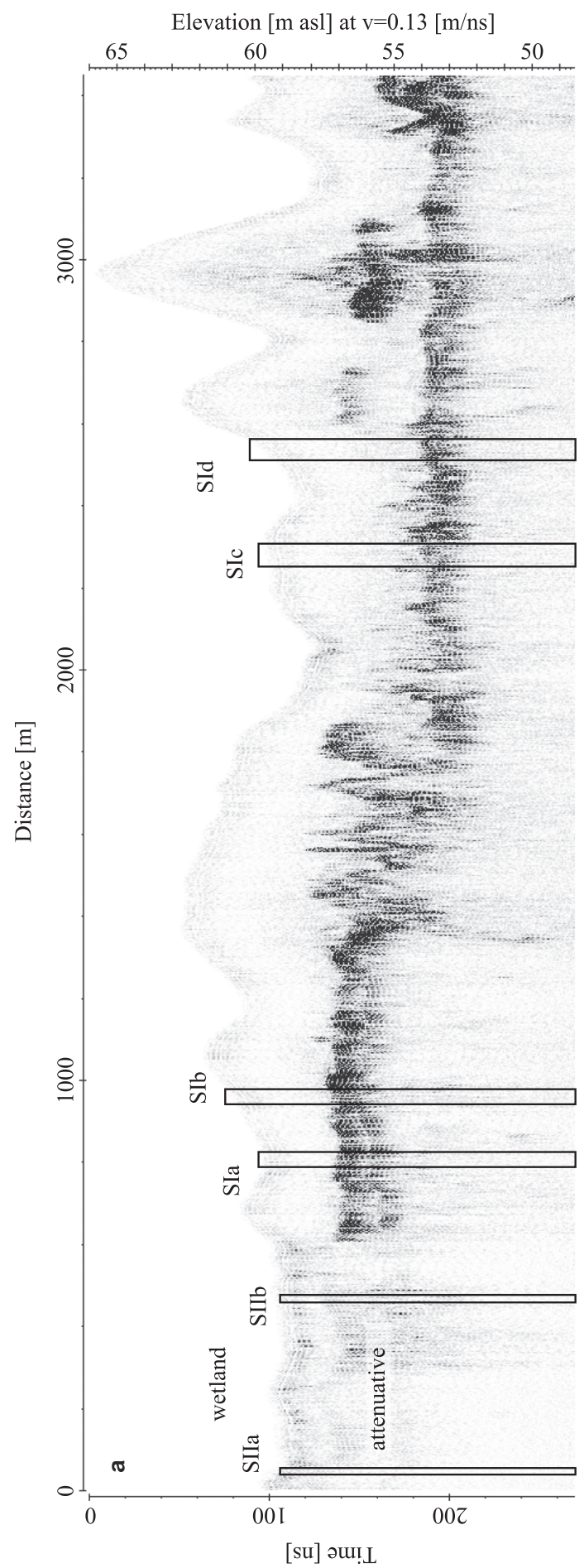

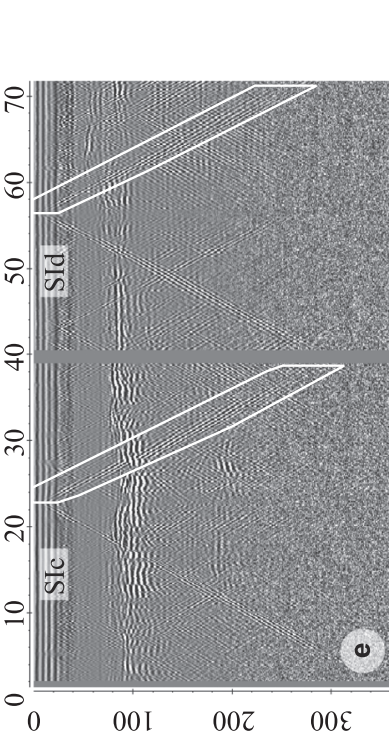

Phase velocity $[\mathrm{m} / \mathrm{ns}]$
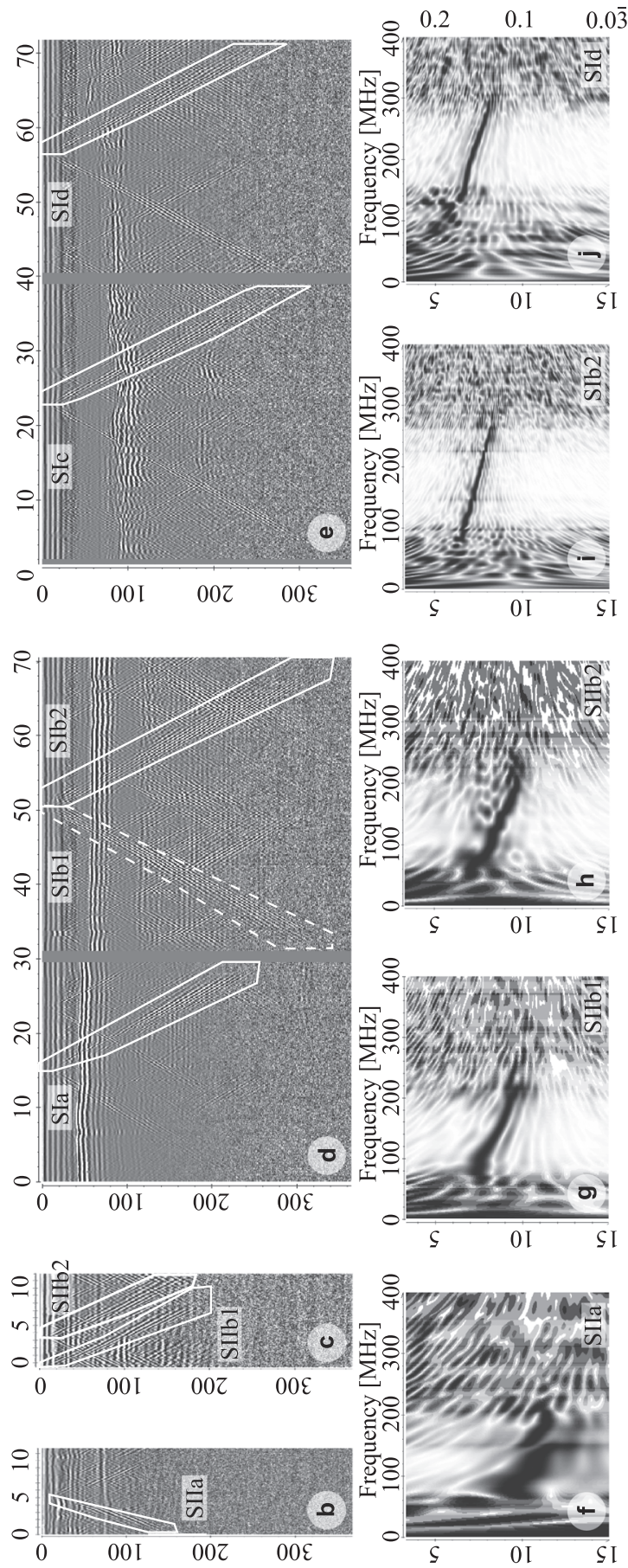

$[\mathrm{w} / \mathrm{su}]$ ssəuмо[s әseчd

Figure 4. Radargram with corrected topography (a) for the common offset field example from the September 2011 survey (north Yeal). (b)-(e) Boxes indicate locations of extracted sections with dispersive diffractions. Diffractions are labeled SIa-d (clean sand) and SIIa-b (wetland). (f)-(i) Examples of dispersion images. Note the attenuation in Figure 4a associated with the wetland at $0-600 \mathrm{~m}$ and the difference in dispersion images (SI vs. SII).

with $v_{f k}=2 f / k$. The $f k$-spectrum was calculated from radar sections without muting. The $f k$-analysis offers the potential for semi-automated extraction of dispersion curve without the need to mute data. This method could be used for larger-scale mapping. The size of the analyzed window provides a method of averaging over larger radar cross-sections.

\subsection{Waveguide Dispersion: May Versus September}

[18] The 250 and $500 \mathrm{MHz}$ data from May 2011 present similar dispersion images (Figures $5 \mathrm{~b}$ and $5 \mathrm{~d}$ ). The $250 \mathrm{MHz}$ data tend to have better signal-to-noise ratio and a thinner dispersion curve indicating higher slowness resolution. We 

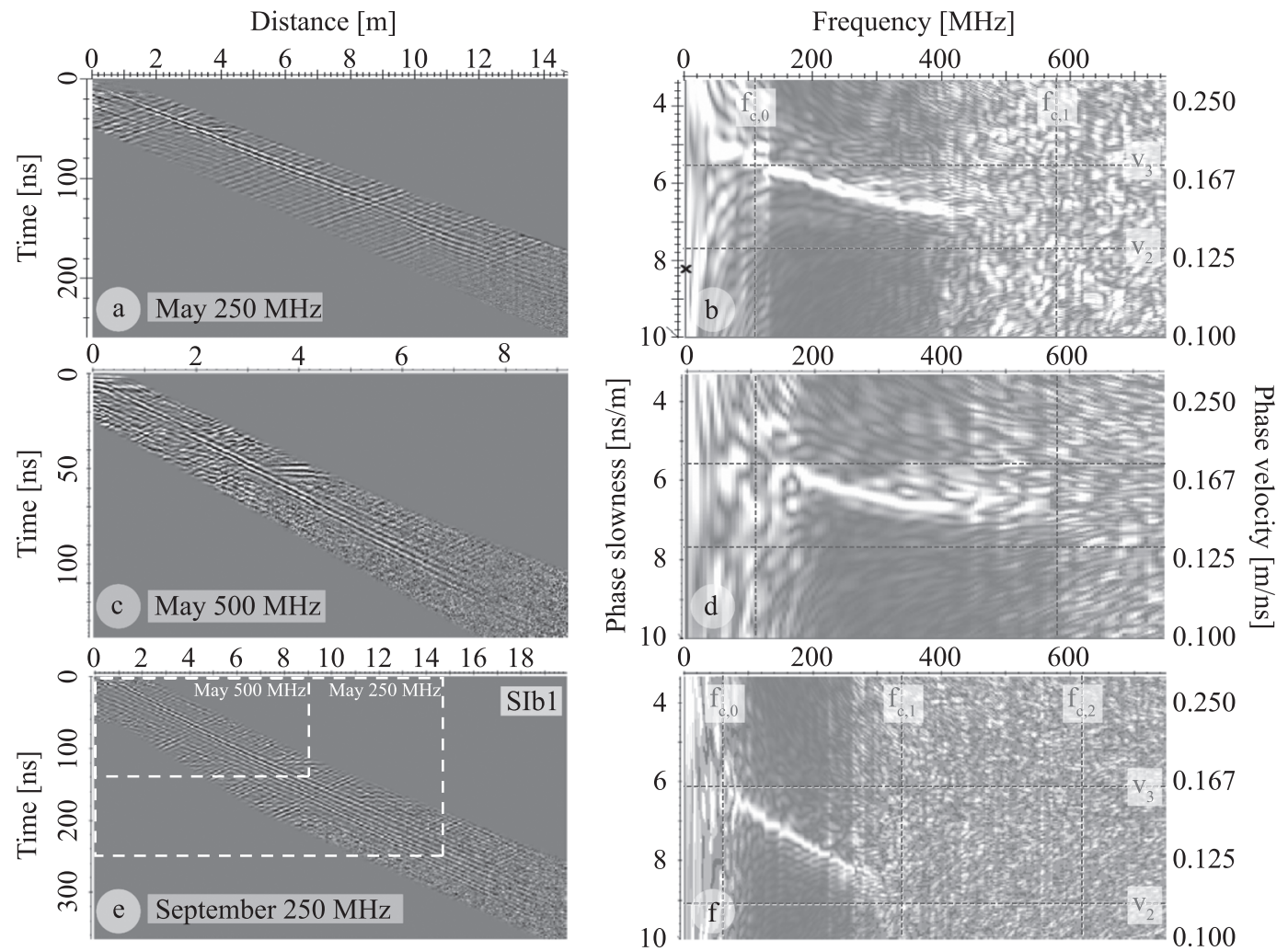

Figure 5. Field $\mathrm{CO}$ gathers from (a) May $250 \mathrm{MHz}$ (white box in Figure 1), (c) May $500 \mathrm{MHz}$, and (e) September $250 \mathrm{MHz}$ (SIb1), and (b, d, f) corresponding dispersion images; cutoff frequencies and upperand lower-layer velocities calculated from model results are shown as dashed lines. Note the different scales in Figures 5a, 5c, 5e. Dashed white boxes in Figure 5e indicate the data extent in Figures 5a and 5c.

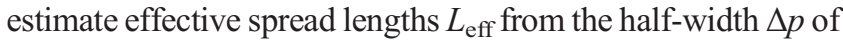
the slowness maxima at several frequencies and obtain values between 18 and $24 \mathrm{~m}$ for the $250 \mathrm{MHz}$ data, and between 8 and $17 \mathrm{~m}$ for the $500 \mathrm{MHz}$ data. The maximum offset at which diffracted energy appears above the noise floor for $\mathrm{CO}$ data is half the effective spread length. This results in good correlation between the maximum distance at which diffraction energy was observed in the field data (i.e., 8 and
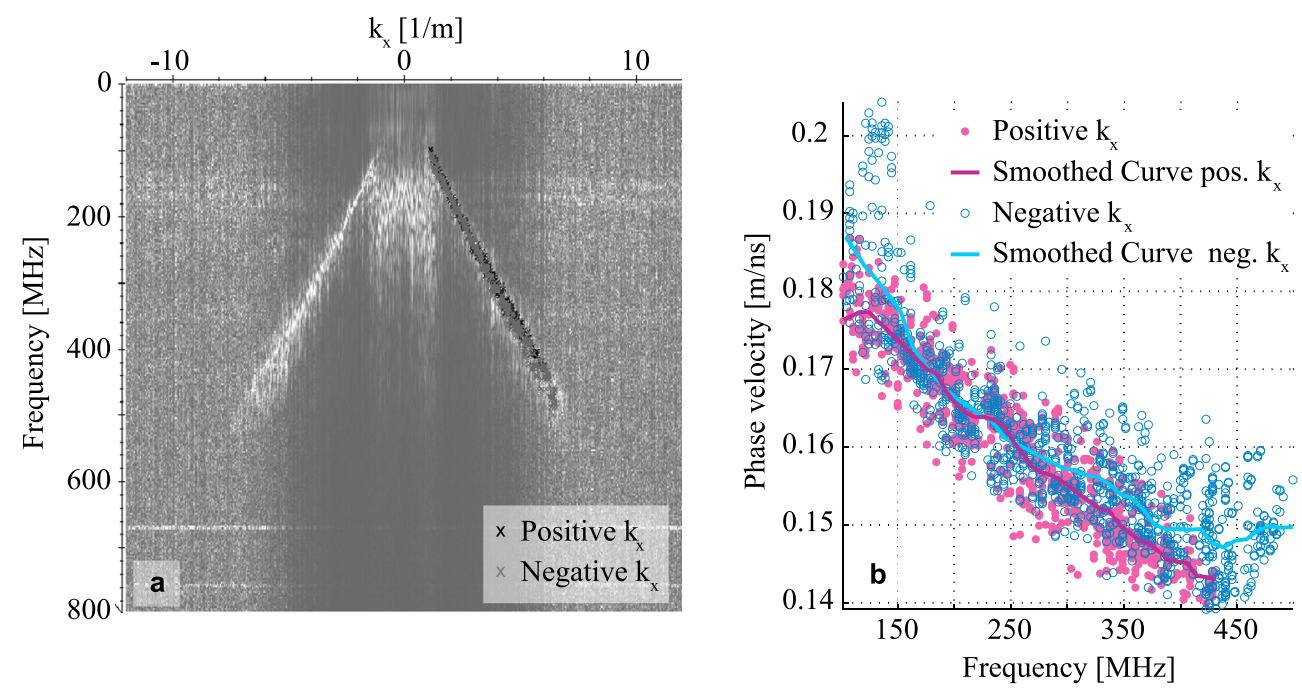

Figure 6. $f k$-spectrum of the entire $250 \mathrm{MHz}$ profile (May 2011, $100 \mathrm{~m}$ ) shows (a) energy bands associated with dispersive diffractions. (b) Phase velocity versus frequency plot, symbols denote values obtained for automatic picking results (i.e., $f k$-spectrum maxima), in magenta for positive and in blue for negative wavenumbers (i.e., black and gray crosses in Figure 6a, respectively). Solid curves in Figure 6b are calculated with an averaging operator and downsampling by interpolation. 


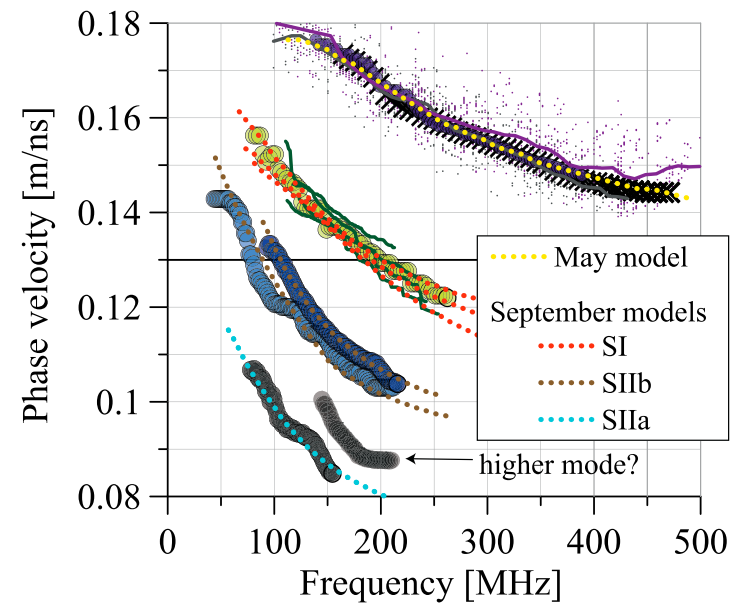

- May (250 MHz)
$\times$ May (500 MHz)
- May fk- picks (neg)
- May fk- smoothed (neg)
— May fk-picks (pos)
May fk- smoothed (pos)

○ September SIb1

- September SIa-d

- Sept. SIIb1

O Sept. SIIb2

- Sept. SIIa

Figure 7. Dispersion curves derived from field data (colored symbols and solid lines), and predicted curves using the model results of inversion (dotted lines) given in Tables 2 and 3. Note the great difference in dispersion characteristic from different date and location.

$15 \mathrm{~m}$ for 500 and $250 \mathrm{MHz}$, respectively) and the predicted spread length from dispersion slowness resolution.

[19] The picked dispersion curves from May are shown in Figure 7. Their velocities range between $0.143 \mathrm{~m} / \mathrm{ns}$ and $0.176 \mathrm{~m} / \mathrm{ns}$ for frequencies between 130 and $450 \mathrm{MHz}$.

[20] To examine the method of picking dispersion curves from the $f k$-spectrum, we transformed the $100 \mathrm{~m}$ long $250 \mathrm{MHz}$ radar section into the frequency-wavenumber domain without muting (Figure 6a). The diffractions appear in the $f k$-spectrum as clouds of energy with a linear slope. Positive and negative wavenumbers represent the left and right diffraction legs, respectively. The resolution of those energy clouds is poor and a continuous curve is not evident. Automatic picking of $f k$-maxima as facilitated in ReflexW reveals clouds of data points after transformation to the velocityfrequency domain (Figures $6 \mathrm{~b}$ and 7 ). Diffraction curves were extracted from those clouds by moving average filtering and interpolating to downsampled and regularly spaced frequency points. Note, however, that the continuity of the diffracted energy in the $f k$-spectrum can be increased by muting unwanted data. This results in dispersion curves that are highly comparable to those picked from the frequency-slowness domain dispersion images.

[21] Dispersion images resulting from our type SI diffractors within the September $250 \mathrm{MHz}$ data show a slightly increased effective spread length between 22 and $35 \mathrm{~m}$ compared to the May data. Their dispersion characteristics are plotted in Figure 7 and reveal velocities between $0.12 \mathrm{~m} / \mathrm{ns}$ and $0.155 \mathrm{~m} / \mathrm{ns}$. The frequency range where a dispersion curve can be identified is between approximately 70 and $260 \mathrm{MHz}$. For a similar frequency range, the SII diffractions in the wetland area show lower velocities of between $0.085 \mathrm{~m} / \mathrm{ns}$ and $0.107 \mathrm{~m} / \mathrm{ns}$ for SIIa and between
$0.102 \mathrm{~m} / \mathrm{ns}$ and $0.145 \mathrm{~m} / \mathrm{ns}$ for SIIb. Their resolution is generally decreased consistent with the maximum distance at which diffracted energy is above the noise floor (see Figures $4 \mathrm{f}-4 \mathrm{~h}$ ). The SIIa dispersion image appears to contain two dispersion curves. The first dispersive energy band ranges between 75 and $155 \mathrm{MHz}$, and the second curve lies at frequencies from 140 to $210 \mathrm{MHz}$.

\subsection{Waveguide Parameter Inversion}

[22] We have applied inversion methods to recover waveguide parameters from our field data. The code we used is that developed by Jan Van der Kruk [Van der Kruk, 2006; Van der Kruk et al., 2006]. Waveguide parameters are recovered from the field experiments by minimizing the misfit between forward modeled and picked dispersion curves using a combined local and global optimization. The global optimization is based on several iterations of local minimization probing a variety of starting models (simplex search method). To test a large parameter space and obtain a better understanding on the model convergence, we performed the global optimization twice. The first optimization covered a coarsely sampled but larger parameter space. The objective was to find constraints for starting values for the second optimization. Results of the inversion process are shown in Figure 8 for the second optimization. The cost function that is to be minimized is defined as the L1-norm. The forward model is based on modal wave propagation [Arcone, 1984; Arcone et al., 2003; Van der Kruk, 2006]. All measured dispersion curves were assumed to represent the fundamental TE mode $\left(\mathrm{TE}_{0}\right)$, except for the higher frequency dispersion curve in SIIa, which was inverted as the first higher mode $\left(\mathrm{TE}_{1}\right)$. Table 2 lists the parameters derived from the curve matching.

\subsection{Inversion Results}

[23] This section summarizes the modeling results from inversion, which are listed in Table 2. In May, a waveguide of $0.2 \mathrm{~m}$ thickness and relative dielectric permittivity values of above 5 (i.e., $\varepsilon_{2, \text { May }} \approx 5.3$ ) and for the half-space below 3 (i.e., $\varepsilon_{3, \text { May }} \approx 2.8$ ) provided a suitable fit between field and model data. According to the complex refractive index model (CRIM) for a quartz sand with $40 \%$ porosity and relative dielectric permittivity for quartz of 4.4 , those values correspond to volumetric water contents [v\%] of $8 \mathrm{v} \%$ and $0.2 \mathrm{v} \%$ for waveguide layer and lower half-space, respectively. The field and model dispersion curves are shown in Figure 7. Inversion results for dispersion curves derived from $f k$-clouds and smoothed curves are almost identical. That means that our method of smoothing and interpolating phase velocity versus frequency picks represents the trend within the data cloud well. Inversion results for positive and negative wavenumbers (right and left diffractions), however, differ slightly. That is expected because the extracted dispersion curves reveal a different trend. While the positive $(f k+)$ dispersion curve is similar to the curves picked from the dispersion image, the negative $(f k-)$ curve probably includes outliers at the low and high frequency end.

[24] The type SI diffractions from the September 2011 data generally predict lower layer velocities compared to May, while the waveguide thickness varied between 0.1 and $0.25 \mathrm{~m}$ (i.e., $h_{\text {SI. }}=0.1 \ldots 0.2 \mathrm{~m}$ ). Relative dielectric 

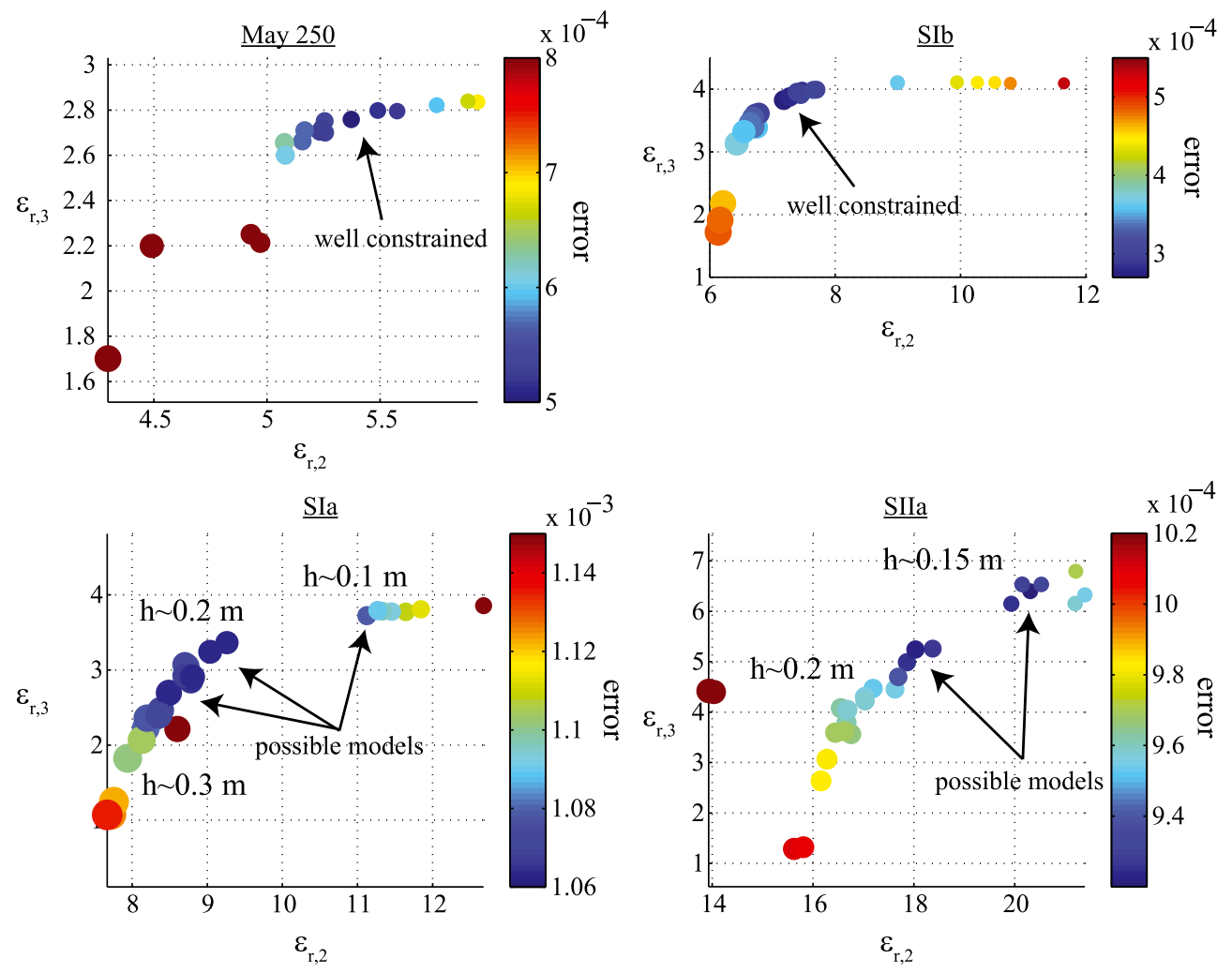

Figure 8. Inversion $L 1$ misfit (blue low, red high values) between model and observed dispersion curves shown as a function of waveguide parameters $\varepsilon_{2}, \varepsilon_{3}$. Circle size indicates waveguide height $h$. Arrows indicate inversion results with low misfit for different sets of parameters illustrating nonuniqueness.

permittivities for waveguide layer were around 7.5 for a $0.25 \mathrm{~m}$ thick waveguide, and between 8.5 and 11 for the thinner waveguide prediction $\left(\varepsilon_{2, \mathrm{SI}}=7.5 \ldots 11\right)$. Models with lower half-space permittivities around 3 (thicker waveguide) and 4 (thinner waveguide) resulted in a good fit with the observed dispersion curves (i.e., $\varepsilon_{3, \mathrm{SI}}=3 \ldots 4.1$ ). Those values correspond to volumetric water contents of approximately $13-20 \mathrm{v} \%$ and $2-5 \mathrm{v} \%$ for waveguide layer and lower half-space, respectively. The possible SIIb models predict a waveguide with similar dielectric permittivities as the SI model with smaller waveguide height. Waveguide permittivities are around 12 (i.e. $\varepsilon_{2, \mathrm{SIIb}}=12.0 \ldots 12.8$ ) and half-space permittivities were similar to the SI values (i.e., $\varepsilon_{3, \mathrm{SIIb}}=3.3$ 4.0 ) for a waveguide of around $0.2 \mathrm{~m}$ thickness (i.e., $h_{\mathrm{SIIb}}=$ $0.2 \mathrm{~m}$ ). Dispersion curves from SIIa diffractions result in the highest dielectric permittivity model predictions for both, waveguide and half-space. The possible models are a waveguide with a height of $0.2 \mathrm{~m}$ when dielectric permittivities are 17.9 and 5 for waveguide and half-space, respectively (i.e., model 1: $\varepsilon_{2, \text { SIIa }}=17.9, \varepsilon_{3, \text { SIIa }}=5.0, h=0.22 \mathrm{~m}$ ). The alternative model is a thinner waveguide of $0.16 \mathrm{~m}$ thickness, and layer permittivities of 20.3 and 6.4 for waveguide and half-space, respectively (i.e., model $2: \varepsilon_{2, \text { SIIa }}=20.3, \varepsilon_{3, \text { SIIa }}=$ $6.4, h=0.16 \mathrm{~m})$. Water contents inferred for the waveguide are high (i.e., above $35 \mathrm{v} \%$ ). Separate analysis of the possible higher-order mode (i.e., $\mathrm{TE}_{1}$ inversion) also resulted in a nonunique solution with models predicting waveguide layer permittivities close to 17 , and half-space permittivites of 2.2 or 7.2 .
[25] Note that the theoretical cutoff frequencies calculated using equation (1), which correspond to May and September models, are reported in Tables 2 and 3 and are displayed as vertical lines in Figures 5b, 5d, and 5e. The first higher mode (i.e., $\mathrm{TE}_{1}$ ) in the May data is predicted at $580 \mathrm{MHz}$. This falls outside of the bandwidth of the antennas used for the measurement and hence this higher mode is not evident in the dispersion images of the common offset data sets. The critical frequency for the $\mathrm{TE}_{1}$-mode in the September SI models is above $330 \mathrm{MHz}$. The dispersion images, however, do not show evidence of that higher mode.

\subsection{Discussion of Inversion Procedure}

[26] Key inputs for effective inversion of waveguide dispersion data are the range and density of frequencies selected [Bikowski et al., 2012]. We have presented inversion results based on best fit to all data points that could reasonably be extracted from the field experiments (see Figure 7). The number of phase velocity frequency data pairs was between 50 and 130 (i.e., as picked from the dispersion images), and around 800 for the $f k$-cloud data. Thus, the frequency range that produced the final inversion result is that of the data shown in Figure 7 and discussed in the previous section. Figure 8 shows inversion results for $\varepsilon_{2}$ plotted against $\varepsilon_{3}$ in a scatter plot where the color of the symbol represents $L 1$ error and the symbol size is proportional to the waveguide height $h$. The inversion for May data seems to be well constrained and we did not encounter nonuniqueness issues within the parameter space used for inversion. 
Table 2. Waveguide and Underlying Half-Space Parameters and Theoretical Cutoff Frequencies $f_{\mathrm{c}, 0}$ and $f_{\mathrm{c}, 1}$ of Fundamental and First Higher Mode, Respectively, Listed for a Selection of Field Data Dispersion Curves Obtained by Inversion [Van der Kruk, 2006]. The $L 1$ Value Represents the Misfit Between Modeled and Measured Dispersion Curves. Dispersion Curves From May Extracted From $250 \mathrm{MHz}$ Data Were Compared Using a Slant Stack (May 250) [Park et al., 1998], and Using fk-method. The Automated Picking in $f k$ Domain Resulted in Velocity Versus Frequency Clouds (i.e., fk-cloud) That Were Smoothed Using Averaging and Interpolation ( $f k$-Curve) for Positive $(+)$ and Negative (-) Wavenumbers Independently (see Figure 7)

\begin{tabular}{lrccccc}
\hline & $\varepsilon_{2}$ & $\varepsilon_{3}$ & $h[\mathrm{~m}]$ & $\mathrm{L} 1$ & $f_{\mathrm{c}, 0}$ & $f_{\mathrm{c}, 1}$ \\
\hline \multirow{2}{*}{ May 250 } & & & May & & & \\
May 500 & 5.4 & 2.8 & 0.20 & $5.02 \mathrm{E}-04$ & 103 & 568 \\
fk-cloud + & 5.3 & 2.7 & 0.21 & $5.20 \mathrm{E}-04$ & 96 & 539 \\
fk-curve + & 6.3 & 2.9 & 0.13 & $3.14 \mathrm{E}-03$ & 128 & 754 \\
fk-cloud - & 4.0 & 2.9 & 0.15 & $6.67 \mathrm{E}-04$ & 120 & 688 \\
fk-curve - & 4.6 & 2.5 & 0.31 & $4.37 \mathrm{E}-03$ & 75 & 409 \\
& & & 0.31 & $1.20 \mathrm{E}-03$ & 79 & 422 \\
& & & & & & \\
SIa & 8.5 & 2.7 & 0.22 & $1.06 \mathrm{E}-03$ & 45 & 328 \\
& 11.1 & 3.7 & 0.12 & $1.08 \mathrm{E}-03$ & 79 & 539 \\
SIb1 & 7.3 & 3.3 & 0.26 & $8.04 \mathrm{E}-04$ & 60 & 348 \\
& 8.6 & 3.8 & 0.19 & $7.17 \mathrm{E}-04$ & 75 & 435 \\
SIb2 & 7.2 & 3.8 & 0.25 & $2.73 \mathrm{E}-04$ & 76 & 402 \\
& 9.9 & 4.1 & 0.12 & $4.32 \mathrm{E}-04$ & 104 & 623 \\
SIc & 9.2 & 3.0 & 0.20 & $7.74 \mathrm{E}-04$ & 50 & 351 \\
& 12.3 & 4.1 & 0.10 & $4.83 \mathrm{E}-04$ & 92 & 616 \\
SIIa TE & 20.3 & 6.4 & 0.16 & $9.20 \mathrm{E}-04$ & 45 & 296 \\
& 17.9 & 5.0 & 0.22 & $9.25 \mathrm{E}-04$ & 31 & 221 \\
SIIa TE & 16.8 & 7.2 & 0.54 & $7.82 \mathrm{E}-04$ & 19 & 109 \\
& 16.7 & 2.2 & 0.56 & $7.29 \mathrm{E}-04$ & 6 & 77 \\
SIIb1 & 12.8 & 4.3 & 0.20 & $1.20 \mathrm{E}-03$ & 46 & 303 \\
SIIb2 & 12.0 & 3.3 & 0.21 & $4.09 \mathrm{E}-04$ & 37 & 279 \\
\hline & & & & & &
\end{tabular}

We found, however, that minimization for some diffractions in September resulted in two models that both minimize the cost function to a sufficient degree as illustrated in Figure 8 (i.e., SIa and SIIa models). Two physically reasonable waveguide geometries result in low misfit (blue symbols). The waveguides differ in dielectric permittivity and waveguide height. The two waveguide constellations that fit the data are (i) a thin waveguide with high dielectric permittivities of waveguide layer and half-space, which corresponds to (ii) a thicker waveguide with lower layer and half-space dielectric permittivities. Although both models can produce dispersion curves with similar shape, this ambiguity is frequency limited. The lower limit is the higher cutoff frequency, which is typically determined by the thinner waveguide model (see Table 2). At higher frequencies, the $\mathrm{TE}_{0}$ dispersion curves will diverge as each model converges to the velocity given by the half-space below the waveguide. The second difference between the two models is the incidence of the first higher mode, which also is at higher frequencies for the thinner waveguide. For the SI data, the thinner waveguide predicts a higher mode at around $350 \mathrm{MHz}$ and the thicker waveguide between 450 and $600 \mathrm{MHz}$. The dispersion images SI of the September field data, however, do not show any signs of a higher mode and the signalto-noise ratio at above approximately $300 \mathrm{MHz}$ appears too low for signal detection.

[27] In a recent publication, Bikowski et al. [2012] investigate the influence of frequency range used for dispersion inversion on recovered parameter certainty. They find that by removing phase velocity data points at the lower frequency end, the lower half-space permittivity result becomes uncertain. By removing data points at the high frequency end, however, the certainty of waveguide layer permittivity and height is affected. Those findings suggest that dispersion curves from May are better defined for their waveguide permittivity and height due to high frequency information, which is consistent with the findings here. Van der Kruk [2006] and Strobbia and Cassiani [2007] also found a lack of sensitivity and nonuniqueness related to waveguide height and dielectric properties in their inversion results.

[28] The SIIa curve shows a second mode in the dispersion image (Figure 4f), but it is not clear that this is a higher-order mode. A joint inversion using the lower frequency part as $\mathrm{TE}_{0}$ and the higher frequency part as $\mathrm{TE}_{1}$ did not decrease the misfit and the predicted model is unrealistic with dielectric permittivity values below 1 (local minimization is unconstrained) and a waveguide height of $2 \mathrm{~m}$ (results not shown here). An alternative explanation for the second mode is disturbance introduced by interface roughness (compare Van der Kruk et al. [2012]) or lateral changes in waveguide properties.

[29] Cutoff frequency prediction for fundamental and higher order modes might be useful to qualitatively interpret inversion results. One might favor the models that predict higher cutoff frequencies (thinner waveguide) as we could not observe higher modes in the dispersion images.

[30] A priori knowledge can provide further constraints for the parameter space used for inversion. In an inhomogeneous geologic environment and at large scales, however, additional information might be difficult to obtain. Certainty and uniqueness could be improved by introducing the frequency-slowness magnitude (i.e., amplitude) of dispersive modes into the inversion process as already proposed by Van der Kruk et al. [2006].

\subsection{Effect of the Rainfall on Waveguide Properties}

[31] We have interpreted the waveguide analysis and inversion results and summarized key outcomes in Table 3. The soil moisture derived from the May 2011 data is typical

Table 3. Waveguide and Underlying Half-Space Parameters and Volumetric Water Content $c_{\mathrm{H} 20}$ for Inverted Dispersion Curves Shown in Figure 7. Model Parameters are Derived Using Inversion of Fundamental TE Mode for Dispersion Curves Extracted From the Field Examples May and September

\begin{tabular}{|c|c|c|c|c|}
\hline & $v[\mathrm{~m} / \mathrm{ns}]$ & $\varepsilon_{r}$ & $h[\mathrm{~m}]$ & $c_{\mathrm{H} 2 \mathrm{O}}{ }^{\mathrm{a}}[\mathrm{v} \%]$ \\
\hline Layer 1 & 0.300 & 1 & 2.00 & - \\
\hline May, layer 2 & 0.130 & 5.3 & 0.20 & 8.1 \\
\hline May, layer 3 & 0.179 & 2.8 & - & 0.2 \\
\hline$f_{\mathrm{c}, 0}: 106$ & & $f_{\mathrm{c}, 1}: 581$ & & \\
\hline Sep. SI, layer 2 & 0.110 & 7.5 & 0.26 & 13.6 \\
\hline Sep. SI, layer3 & 0.165 & 3.3 & - & 2.0 \\
\hline$f_{\mathrm{c}, 0}: 57$ & & $f_{\mathrm{c}, 1}: 339$ & & \\
\hline Sep. SIIb2, layer 2 & 0.087 & 12 & 0.21 & 22.7 \\
\hline Sep. SIIb2, layer3 & 0.165 & 3.3 & - & 2.0 \\
\hline$f_{\mathrm{c}, 0}: 37$ & & $f_{\mathrm{c}, 1}: 279$ & & \\
\hline Sep. SIIa, layer 2 & 0.067 & 20.3 & 0.16 & 35.8 \\
\hline Sep. SIIa, layer3 & 0.119 & 6.4 & - & 11.0 \\
\hline$f_{\mathrm{c}, 0}: 45$ & & $f_{\mathrm{c}, 1}: 296$ & & \\
\hline
\end{tabular}

${ }^{\mathrm{a}}$ Estimated with CRIM model (porosity $0.4, \varepsilon_{r, \mathrm{~s}} 4.4$ ). 
for the period immediately after early precipitation in sandy environments following an otherwise extended dry summer period. The uppermost layer is moist sand underlain by dry sand. Toward the end of the wet season in September, the dispersion characteristics changed significantly for the sand part of the profile (SI diffractions). The waveguide again occurred after extensive rain, which was preceded by a period of dry conditions. Intermittent rain persisted during data acquisition. When compared to the May 2011 results, the soil moisture in the waveguide is higher and the sand below is slightly moist rather than dry. The derived dielectric permittivity for the lower half-space in the thick SI models appears quite low (i.e., $\varepsilon_{3, \mathrm{SI} 1} \approx 3.3, v=0.165 \mathrm{~m} / \mathrm{ns}, c_{\mathrm{H} 2 \mathrm{O}} \approx 2 \%$ ) and more realistic for the thin waveguide model (i.e., $\varepsilon_{3, \text { SI } 2} \approx 4$, $v=0.15 \mathrm{~m} / \mathrm{ns}, c_{\mathrm{H} 2 \mathrm{O}} \approx 5 \%$ ). Diffractions found on wetland soils reveal the highest water content. In particular diffraction SIIa predicts volumetric water contents between 0.35 and 0.4 , depending on the choice of model and porosity used for the CRIM. The peat-like surface layer is very rich in organics and may have a porosity of up to $60 \%$. It is likely that this material can take up and temporarily hold large amounts of water. Here, porosity is expected to be much larger than other mineral soils and high water content is unlikely to equate to full saturation of these more peaty soils.

[32] The velocities and moisture contents derived from waveguide properties are reasonable within the framework of other field observations made on the Gnangara Mound [Strobach et al., 2012a, 2012b, and references therein]. That is, in May 2011 high velocities above $0.17 \mathrm{~m} / \mathrm{ns}$ are common for the extremely dry sands down to several meters.

\section{Conclusions}

[33] A high dielectric permittivity layer embedded into lower dielectric layers above and below leads to a waveguide for radiated electromagnetic energy (i.e., radar waves). These trapped waves travel and disperse within the shallow low-velocity waveguide with very low loss. Heterogeneities within the shallow waveguide produce diffractions in ground-penetrating radar data. In common offset radargrams, this diffracted energy appears as straight lines with dispersive pattern. The dispersion characteristics of those diffracted events contain the same dispersion information as the direct waves sampled with a common midpoint or common shotpoint geometry. The linear moveout of common offset diffractions, however, is twice the slope and spatial aliasing therefore occurs at lower frequencies compared to the common shot geometry for a given spatial sample interval. The dispersiveness of the common offset diffracted wavefields is consistent with two-way travel, which means that the diffractor we investigated scatters an already dispersive wavefield. Hence, the resolution of maxima in common offset dispersion images is higher compared to resolution obtained from a common shot gather with equivalent offset. Thus, after two-way time adjustment, a multioffset data analysis method can be used on common offset data to compute dielectric properties of the near subsurface.

[34] Common offset diffraction hyperbolae from two field campaigns have been analyzed for their dispersion characteristics. To extract dispersion characteristics, two methods have been compared. The slant-stack method provides frequency-slowness dispersion images, which reveal high resolution after extracting single diffracting legs and muting unwanted data. For rapid dispersion characterization, a data transformation to frequency-wavenumber domain can offer an alternative method that does not require muting and offers adjustable spatial averaging. Preliminary results validate this approach.

[35] We successfully recover waveguide parameters from our field data by inversion, using modal wave propagation in a single-layer waveguide model. Waveguide velocities can be linked to dielectric permittivity and layer water content. The examples illustrate the sensitivity of this method to subtle variations in waveguide properties due to seasonal changes in water content and lateral variations due to different soil material. The seasonal changes in waveguide dielectric properties are attributed to water content variations related to rainfall. Lateral variations are likely due to differences in water retention properties of the surface soil horizon. With this new interpretation scheme, soil moisture distribution can be derived for the shallow subsurface on large scales.

[36] Shallow diffractions in GPR data are common in near-surface settings and can have many origins. Suitable diffractions may result from tree roots with high dielectric permittivity or dry pockets within partially water-saturated sands. The dispersive characteristics of diffractions in shallow waveguides have been demonstrated as a new way of extracting information from common offset ground-penetrating radar data - the most commonly acquired data. We see many potential scientific applications for our work. Possible applications include estimation of shallow moisture distribution for example during infiltration experiments, soil characterization in agriculture, monitoring of surficial thawing of permafrost, early target identification in unexploded ordnance (UXO) detection and terrestrial or extraterrestrial pedological investigations.

[37] Acknowledgments. We would like to thank Eva Caspari, Tobias M. Müller and Roman Pevzner for their constructive discussions. Jan Van der Kruk kindly provided his code for waveguide inversion. Andrew Greenwood and Penny Wallace-Bell assisted with the data collection in the field. Boris Gurevich greatly improved the original manuscript. We thank Anthony Endres, Jan van der Kruk, and one anonymous reviewer for their constructive comments that lead to improvement and enhancement of the original work. Elmar Strobach is supported by a CIPRS scholarship through Curtin University and an ASEG research foundation grant. We appreciate the ongoing support from both the Water Corporation and Department of Water of Western Australia, who make significant contributions to hydrogeophysical research and development.

\section{References}

Arcone, S. (1984), Field observations of electromagnetic pulse propagation in dielectric slabs, Geophysics, 49, 1763.

Arcone, S., P. Peapples, and L. Liu (2003), Propagation of a groundpenetrating radar (GPR) pulse in a thin-surface waveguide, Geophysics, 68(6), 1922.

Busch, S., J. Van der Kruk, J. Bikowski, and H. Vereecken (2012), Quantitative conductivity and permittivity estimation using full-waveform inversion of on-ground GPR data, Geophysics, 77, H79-H91.

Bikowski, J., J. A. Huisman, J. A. Vrugt, H. Vereecken and J. van der Kruk (2012), Integrated analysis of waveguide dispersed GPR pulses using deterministic and bayesian inversion methods, Near Surface Geophysics, in press, available online, doi:10.3997/1873-0604.2012041.

Cassiani, G., C. Strobbia, M. Giustiniani, N. Fusi, G. B. Crosta, P. Frattini (2006), Monitoring of hydrological hillslope processes via time-lapse ground-penetrating radar, Bollettino di Geofisica Teorica ed Applicata, 47(1-2), 125-144. 


\section{STROBACH ET AL.: WAVEGUIDE PROPERTIES FROM SHALLOW DIFFRACTIONS IN COMMON OFFSET GPR}

Chen, C. C. (2012), Lateral waves in ground penetrating radar applications, 14th International Conference on Ground Penetrating Radar (GPR), Shanghai, China, pp. 39-41.

Davidson, W. A. (1995), Hydrogeology and groundwater resources of the Perth Region Western Australia, Geol. Surv. of Western Aust. Bulletin 142, Western Australian Geological Survey, Perth.

Forbriger, T. (2003), Inversion of shallow-seismic wavefields: I. wavefield transformation, Geophysical Journal International, 153(3), 719-734.

Grote, K., C. Anger, B. Kelly, S. Hubbard, and Y. Rubin (2010), Characterization of soil water content variability and soil texture using GPR groundwave techniques, Journal of Environmental \& Engineering Geophysics, 15(3), 93.

Huisman, J. A., S. S. Hubbard, J. D. Redman, and A. P. Annan (2003), Measuring soil water content with ground penetrating radar: A review, Vadose Zone J., 2(4), 476-491, doi: 10.2113/2.4.476.

Lambot, S., E. Slob, M. Vanclooster, and H. Vereecken (2006), Closed loop GPR data inversion for soil hydraulic and electric property determination, Geophys. Res. Lett., 33(21), L21405.

Park, C., R. Miller, J. Xia, et al. (1998), Imaging dispersion curves of surface waves on multi-channel record, Expanded Abstract: Soc. Explor. Geophys, pp. 1377-1380.

Pettinelli, E., G. Vannaroni, B. Di Pasquo, E. Mattei, A. Di Matteo, A. De Santis, and P. Annan (2007), Correlation between near-surface electromagnetic soil parameters and early-time GPR signals: An experimental study, Geophysics, 72(2), A25.

Prankongkep, N., R. J. Gilkes, B. Singh, and S. Wong (2010), The morphology and composition of pyrite in sandy podosols in the Swan Coastal Plain, 19th World Congress of Soil Science, Brisbane, Australia.

Sandmeier, K. J. (2012), ReflexW Version 6.0, Windows ${ }^{\mathrm{TM}}$ 9x/NT/2000/ $\mathrm{XP} / 7$-program for the processing of seismic, acoustic or electromagnetic reflection, refraction and transmission data, Reference Manual.

Steelman, C., A. Endres, and J. Van der Kruk (2010), Field observations of shallow freeze and thaw processes using high-frequency ground-penetrating radar, Hydrol. Processes, 24(14), 2022-2033.
Strobbia, C., and G. Cassiani (2007), Multilayer ground-penetrating radar guided waves in shallow soil layers for estimating soil water content, Geophysics, 72(4), J17-29, doi: 10.1190/1.2716374.

Strobach, E., B. D. Harris, J. Christian Dupuis, A. W. Kepic, and M. W. Martin (2012a), Cross well radar and vertical radar profiling methods for time lapse monitoring of rainfall infiltration, 22nd ASEG Conference Extended Abstracts, Brisbane, Australia, pp. 1-4.

Strobach, E., B. D. Harris, J. C. Dupuis, A. W. Kepic, and M. W. Martin (2012b), Time-lapse borehole radar measurements in a sandy groundwater system during a winter recharge cycle, 14th International Conference on Ground Penetrating Radar (GPR), Shanghai, China, pp. 689-694.

Van der Kruk, J. (2006), Properties of surface waveguides derived from inversion of fundamental and higher mode dispersive GPR data, Geoscience and Remote Sensing IEEE Transactions on, 44(10), 2908-2915.

Van der Kruk, J., R. Streich, and A. G. Green (2006), Properties of surface waveguides derived from separate and joint inversion of dispersive TE and TM GPR data, Geophysics, 71(1), K19-29, doi: 10.1190/1.2168011.

Van der Kruk, J., C. Steelman, A. Endres, and H. Vereecken (2009a), Dispersion inversion of electromagnetic pulse propagation within freezing and thawing soil waveguides, Geophysical Research Letters, 36(18), L18,503.

Van der Kruk, J., Vereecken, H., and R.W. Jacob (2009b), Identifying dispersive GPR signals and inverting for surface wave-guide properties, The Leading Edge, 28, 1234-1239.

Van der Kruk, J., R. W. Jacob, and H. Vereecken (2010), Properties of precipitation-induced multilayer surface waveguides derived from inversion of dispersive TE and TM GPR data, Geophysics, 75(4), WA263-273, doi: $10.1190 / 1.3467444$.

Van der Kruk, J., N. Diamanti, A. Giannopoulos, and H. Vereecken (2012), Inversion of dispersive GPR pulse propagation in waveguides with heterogeneities and rough and dipping interfaces, Journal of applied Geophysics, 81, 88-96. 